\title{
Tradução e validação da versão brasileira do Children's Eating Attitudes Test (ChEAT)
}

\author{
Translation and validation of the Brazilian version of the Children's Eating Attitudes Test (ChEAT)
}

Nádia Pinheiro ${ }^{[a]}$, Manuel Jiménez ${ }^{[b]}$

\footnotetext{
${ }^{[a]}$ Mestre em Psicologia da Saúde, Universidad de Málaga, Málaga - Espanha, e-mail: nadiaprazeres@yahoo.com.br

${ }^{[b]}$ Professor doutor titular do Departamento de Personalidad, Evaluación y Tratamiento Psicológico, Universidad de Málaga, Málaga - Espanha, e-mail: mjiménez@uma.es
}

Recebido: $19 / 11 / 2010$ Received: 11/19/2010

Aprovado: $10 / 03 / 2011$ Approved: 03/10/2011

\section{Resumo}

\begin{abstract}
Analisou-se a estrutura fatorial e propriedades psicométricas da versão brasileira do Children Eating Attitudes Test (ChEAT) e examinaram-se atitudes e comportamentos alimentares problemáticos em crianças da cidade de São Luís, Maranhão. Trezentos e quarenta e sete participantes (160 meninos e 187 meninas) de 8 a 12 anos foram pesados, medidos e completaram o questionário ChEAT. Encontraram-se três fatores para o teste, com alta consistência interna para a escala total e suas subescalas. Encontraram-se diferenças significativas em atitudes e comportamentos alimentares segundo a idade e o Índice de Massa Corporal (IMC), bem como diferenças em cada fator do ChEAT segundo sexo, idade e IMC. Outros resultados foram que $21 \%$ dos participantes apresentam sobrepeso e 5,8\% obesidade; e que $43,5 \%$ atitudes e comportamentos alimentares alterados. O ChEAT é um instrumento que pode ser utilizado na população infantil brasileira detectando que meninos e meninas que desejam um corpo belo, adotam, para isso, atitudes e comportamentos alimentares implicados na etiologia dos transtornos alimentares.
\end{abstract}

Palavras-chave: ChEAT. Validação. Brasil.

\begin{abstract}
The factorial structure and the psychometric properties of the Brazilian version of the Children Eating Attitudes Test (ChEAT) were analyzed and eating attitudes and behaviors were examined. Three hundred, forty-seven children (160 boys and 187 girls), with ages ranging from 8 to 12 years old, were weighed, measured, and completed the ChEAT questionnaire. A three-factor structure was found for ChEAT, with high internal consistency for the entire test and each factor. Significant differences in eating attitudes and behavior according to age group and BMI (Body Mass Index), as well from each ChEAT factor according to sex, age and BMI were found. Other results showed that $21 \%$ of the children are overweight and $5.8 \%$ are obese and that $43.5 \%$ present problematic eating attitudes and behaviors. ChEAT is a good tool that can be used in Brazilian population, detecting that children do worry about having a beautiful body, for that purpose adopting eating behaviors that take part on the genesis of eating disorders.
\end{abstract}

Keywords: ChEAT. Validation. Brazil. 


\section{Introdução}

Os transtornos alimentares estão presentes na infância, sua prevalência está aumentando e, ao mesmo tempo, seu início está tendo lugar em idades cada vez menores. Desde a década de 1980, afirma-se que pré-adolescentes e adolescentes se preocupam com o peso, querem ser mais magros e tentam perder peso (Maloney, McGuire, Daniels \& Specker, 1989). Para conseguir esses objetivos, se implicam em condutas alimentares problemáticas que representam risco à aparição de alterações ou distúrbios alimentares.

Essa conclusão foi sinalizada por investigações que indicam que primeiros indícios de alterações da conduta alimentar ocorrem na infância (Alonso, Rodríguez, Alonso, Carretero \& Martín, 2005; Jones, Bennett, Olmsted, Lawson \& Rodin, 2001; Kelly, Ricciardely \& Clarke, 1999; Schur, Sanders \& Steiner, 2000; Tremblay \& Lariviere, 2009). E que atitudes e comportamentos alimentares alterados estão presentes a graus alarmantes já entre os 7 e 8 anos (Edlund, Halvarsson \& Sjoden, 1996; Rolland, Farnill \& Griffiths, 1997). Entre as atitudes e os comportamentos alterados incluem-se insatisfação corporal; preocupação e ações relacionadas com a prática de dietas; prática de exercícios físicos exagerados para perder peso; episódios de perda de controle diante da ingestão de alimentos (binge eating); prática de purgas com uso de laxantes, diuréticos ou vômitos autoinduzidos; demasiada preocupação com a comida e seu conteúdo calórico, chegando à evitação de determinados alimentos (Jones, Bennet, Olmsted, Lawson \& Rodin, 2001; Kaluski, Natamba, Goldsmith, Shimony \& Berry, 2008; Maloney, McGuire, Daniels \& Specker, 1989; Ricciardelli \& McCabe, 2001a). Esses comportamentos, por sua vez, podem levar ao aparecimento do transtorno alimentar completo (Forman-Hoffman, 2004; Ricciardelli \& McCabe, 2001b; Shisslak, Crago, McKnight, Estes, Gray \& Parnaby, 1998).

Investigações em diversos países do mundo apontam para essa mesma direção. Na Croácia, os pré-adolescentes apresentam alterações moderadas e graves em seus comportamentos alimentares (Knez, Munjas, Petrovecki, Paucic-Kirincic \& Persic, 2006).

Nos Emirados Árabes, 23,4\% da população adolescente estudada apresentava problemas alimentares associados com a interiorização do ideal de magreza e o conhecimento de estratégias de emagrecimento (Eapen, Mabrouk \& Bin-Othman, 2006). Na Suécia, um estudo realizado com meninas de 7 anos, indica que $28 \%$ delas querem emagrecer, $22 \%$ tentam perder peso e $20 \%$ comem menos com o claro objetivo de perder peso (Edlund, Halvarsson \& Sjoden, 1996).

Os comportamentos alimentares problemáticos e sintomas de transtornos alimentares em pré-adolescentes são comumente avaliados com o instrumento Children Eating Attitudes Test (ChEAT; Maloney, McGuire \& Daniels, 1988), que é a versão infantil do Eating Attitudes Test (EAT-26, Garner, Olmsted, Borth \& Garfinkel, 1982).

O ChEAT é uma adaptação do EAT-26, cuja validação e análise de estrutura fatorial aponta para a existência de três fatores: Fazer dieta composto por 13 itens $(1,6,7,10,11,12,14,16,17,22,23,24,25)$ com $\alpha=.90$, Bulimia e Preocupação com a comida, composto por seis itens $(3,4,9,18,21,26)$ com $\alpha=.84$ e Controle Oral, com sete itens $(2,5,8,13,15,19,20)$ e $\alpha=$ .83 (Garner, Olmsted, Borh \& Garfinkel, 1982).

Já o ChEAT foi desenvolvido para avaliar a presença de comportamentos e atitudes alimentares em menores de 15 anos.

Em 1994, Smolak e Levine realizaram uma avaliação psicométrica do ChEAT em uma amostra de 308 meninas. Os resultados foram que a fiabilidade do teste é de .87 , mas elimina-se o item 19 , sobe a .88. Foram encontrados 4 fatores: Fazer dieta (1, $2,6,10,11,12,14,16,17,22,23,24)$, Restrição e purga $(2,7,9,10,17,22,23,24,26)$, Preocupação com a comida $(3,4,18,20,21)$ e Controle oral (8, $13,15,20)$.

Em 2005, um grupo de investigadores de Tarragona conduziram um estudo similar com a versão catalã do questionário (Sancho, Asorey, Arija \& Canals, 2005). Uma vez mais, foram encontrados quatro fatores: Medo e preocupação por engordar, Pressão social para comer, Preocupação com a comida e Restrição alimentar. No entanto, essa versão contém apenas 20 dos 26 itens, já que seis foram considerados inapropriados para a idade.

Lynch e Eppers-Reynolds (2005) propuseram uma estrutura de cinco fatores após aplicar o questionário em 2.093 meninos e meninas: Fazer dieta $(1,23$, 24), Imagem corporal $(1,11,14)$, Preocupação com a comida $(4,18,21)$, Atenção aos elementos constituintes dos alimentos $(6,7,16)$ e Pressão social para comer $(8,20)$. 
Rodas (2006), da Universidade de Málaga, realizou um estudo similar onde se obtiveram 3 fatores que explicam 32,86\% da variança: Preocupação com a comida ( 3 , 18, 21 e 25), Fazer dieta e purga $(1,2,6,7,9,10,11,12$, $14,16,17,22,23,24)$ e Controle oral $(8,13,15,20$ y 26$)$.

Em 2007, um grupo da Universidade de Girona realizou uma validação do ChEAT com 457 participantes de 8 a 12 anos. Encontraram, também, uma estrutura semelhante a estrutura original de três fatores, com alpha de Cronbach de .76 para o teste, .57 para Controle oral, .50 para Bulimia e .74 para Dieta (Gracia, Marcó \& Trujano, 2007).

Por outro lado, Senra, Seoane, Vilas e Sánchez-Cao (2007), após a execução de um estudo similar com uma população de 546 meninos e 530 meninas, encontraram quatro fatores: Medo de engordar, Restrição alimentar, Pressão social para comer e Desinibição.

0 primeiro objetivo deste trabalho foi validar a versão experimental do ChEAT, examinando a estrutura fatorial, consistência interna do questionário e de seus componentes principais encontrados, aplicando-o em uma amostra de crianças brasileiras.

0 segundo objetivo foi verificar a presença de atitudes e condutas alimentares alteradas na população em estudo, ou seja, examinar a pontuação total obtida no teste.

0 terceiro objetivo foi estudar a relação entre os fatores do ChEAT e a pontuação total do ChEAT (atitudes e comportamentos alimentares) com gênero, idade e Índice de Massa Corporal (IMC). Concretamente, avaliar as relações: 1 ) entre gênero e atitudes e comportamentos alimentares problemáticos (pontuação total do ChEAT) para examinar se os meninos obtêm pontuações totais significativamente mais altas que as meninas (Gracia, Marcó \& Trujano, 2007); e entre gênero e cada fator do ChEAT. 2) entre idade e atitudes e comportamentos alimentares problemáticos (pontuação total do ChEAT) para verificar se os mais jovens pontuam mais alto que os mais velhos (Alonso, Rodríguez, Alonso, Carretero \& Martín, 2005; Knez, Munjas, Petrovecki, Paucic-Kirincic \& Persic, 2006; Tremblay \& Lariviere, 2009) ou se a diferença, segundo a idade, não é significativa (Gracia, Marcó \& Trujano, 2007); e entre idade e cada fator do ChEAT. 3) entre IMC e atitudes e comportamentos alimentares problemáticos (pontuação total do ChEAT) para comprovar se quanto mais alto é o IMC, manifestam-se, tais atitudes e comportamentos alterados (Ackard \& Peterson, 2001; Killen et al., 1996); e entre IMC e cada fator do ChEAT.

\section{Método}

\section{Participantes}

Participaram do estudo, 347 pré-adolescentes, de 8 a 12 anos ( $M=10.00$, DT = 1,49), sendo 53,9\% meninas e 46,1\% meninos (Tabela 1), de 4 escolas privadas de São Luís, Maranhão. A participação foi voluntária após a devolução do consentimento livre e esclarecido assinado por um dos pais ou responsável.

Tabela 1 - Distribuição da amostra por gênero e idade

\begin{tabular}{ccccccc}
\hline & \multicolumn{2}{c}{ Total } & \multicolumn{2}{c}{ Meninos } & \multicolumn{2}{c}{ Meninas } \\
\hline & N & \% & N & \% & N & \% \\
\hline Total & 347 & 100 & 160 & 46.1 & 187 & 53.9 \\
\hline anos & 85 & 24.5 & 39 & 24.4 & 46 & 24.6 \\
\hline 9 anos & 51 & 14.7 & 22 & 13.8 & 29 & 15.5 \\
10 anos & 67 & 19.3 & 34 & 21.3 & 33 & 17.6 \\
11 anos & 66 & 19 & 30 & 18.8 & 36 & 19.3 \\
12 anos & 78 & 22.5 & 35 & 21.9 & 43 & 23 \\
\hline
\end{tabular}

Fonte: Dados da pesquisa.

\section{Instrumentos}

Fita métrica e balança digital. Para calcular o Índice de Massa Corporal (IMC), a altura de cada criança foi medida sem sapatos, com uma fita métrica. 0 peso foi medido, sem sapatos em uma balança digital Techline. 0 índice IMC foi calculado com peso/altura $(\mathrm{m})^{2}$ e classificado de acordo com a Tabela Internacional de Crescimento Longitudinal Infantil (Cole, Belizzi, Flegal \& Dietz, 2000).

Children's Eating Attitudes Test (ChEAT). O ChEAT (Maloney, McGuire \& Daniels, 1988) é uma escala de 26 itens que avalia atitudes e condutas alimentares problemáticas, alteradas ou desadaptadas. Cada item é medido em uma escala Likert de seis pontos que varia do um (nunca) a seis (sempre). Os autores, para calcularem a pontuação total, recodificam o questionário a uma escala de quatro pontos: as respostas pontuadas com seis são recodificadas a três, as pontuadas com cinco são recodificadas a dois, e as que variam entre um e três são recodificadas a zero. Em seguida, realizam a soma de todos os itens obtendo a pontuação total (atitudes e comportamentos alterados). 
Uma versão experimental foi utilizada nesta investigação (Anexo A). A versão original foi traduzida ao português. Entretanto, para cumprir o primeiro objetivo, validar o questionário na população infantil brasileira, a escala de seis pontos foi mantida a fim de capturar a variabilidade da escala completa. Essa alternativa foi utilizada em outras validações do ChEAT por Anton et al. (2006) e Lynch e Epper-Reynolds (2005). Já para a análise de atitudes e comportamentos alimentares alterados foi realizada a recodificação já explicada.

\section{Procedimento}

Um contato inicial com os diretores e coordenadores das escolas foi realizado para explicar-lhes os objetivos e procedimentos do estudo. Após a autorização da escola, entregou-se a cada aluno entre 8 e 12 anos, um consentimento livre e esclarecido que deveria ser lido e assinado por um responsável legal, caso a participação fosse autorizada. Aproximadamente $40 \%$ dos consentimentos retornaram.

No dia estabelecido com a coordenação e professorado, um investigador e uma aluna de graduação em Psicologia, previamente treinada, foram às escolas aplicar os instrumentos, sempre afirmando o caráter voluntário e anônimo do trabalho. Os instrumentos eram aplicados em grupos de dez a 20 alunos.

\section{Análise de dados}

Para realizar as análises estatísticas, foi utilizado o programa informático Statistical Package for the Social Sciences (SPSS) versão 17.0.

Para a validação e verificação de estrutura fatorial do ChEAT, foram realizadas duas análises. A primeira, uma redução de dimensões ou análise fatorial de componentes principais com rotação Varimax; a segunda, uma análise de fiabilidade modelo alfa, para o teste completo e para cada fator encontrado.

Para cumprir com o segundo objetivo, o questionário foi recodificado à escala de quatro pontos e estabelecido o ponto de corte de 20 , assim como sugerem os autores do instrumento. Aqueles cuja pontuação total supera os 20 pontos apresentam atitudes e comportamentos alimentares alterados e estão em risco a desenvolver o transtorno alimentar completo.

Para examinar a existência ou não de diferenças em função do gênero em atitudes e comportamentos alimentares alterados (pontuação total do ChEAT) e em cada fator encontrado, realizaram-se dois T-test: um com gênero como variável de agrupação e atitudes e comportamentos alterados como variável para contrastar, e outra com gênero como variável de agrupação e cada fator do ChEAT como variável para contrastar.

Para examinar a existência ou não de diferenças em função da idade, realizou-se ANOVA com idade como fator e atitudes e comportamentos alterados e cada fator do ChEAT como variáveis dependentes. Realizou-se outra ANOVA, desta vez para verificar diferenças em função do IMC (fator) em atitudes e comportamentos alimentares alterados e cada fator do ChEAT (dependentes). Por haver mais de um grupo de idade e mais de um grupo de IMC, realizou-se provas post-hoc.

\section{Resultados}

\section{Estrutura fatorial do ChEAT}

Com um D $=.003$ e KMO = .708, considerou-se oportuno realizar a análise fatorial.

Foram extraídos oito fatores que explicavam $57,62 \%$ da variação total com um alpha de Cronbach aceitável de .673. Resultado semelhante ao encontrado por Smolak e Levine, que em 1994, encontraram sete fatores e por Rodas (2006) que encontrou oito fatores.

Entretanto, após a análise do gráfico de sedimentação e dos autovalores iniciais, apenas três dos oito fatores eram sólidos e explicavam $33,22 \%$ da variação total. Por isso, a análise fatorial foi novamente realizada, fixando-se o número de fatores em três e a supressão dos coeficientes menores que .20 . Verifica-se que o coeficiente de fiabilidade do questionário aumenta com a eliminação dos itens cinco e 19, chegando a .691 . 0 resultado está exposto na Tabela 2.

Tabela 2 - Fatores encontrados na versão brasileira do ChEAT

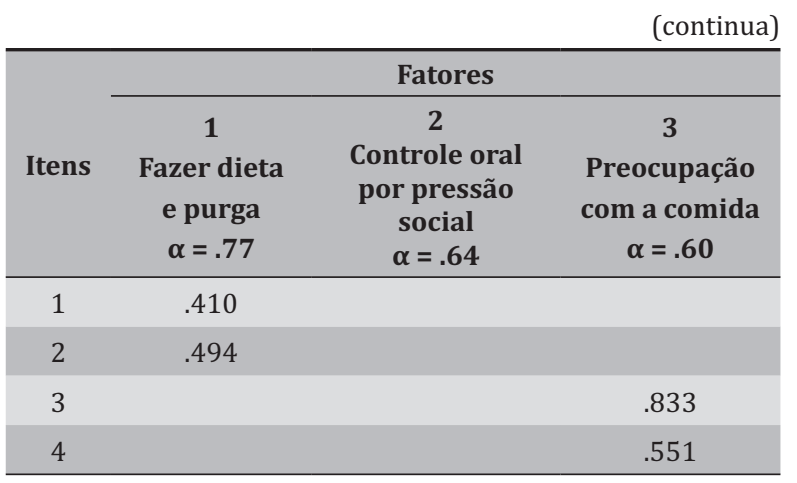


Tabela 2 - Fatores encontrados na versão brasileira do ChEAT

\begin{tabular}{|c|c|c|c|}
\hline \multirow[b]{2}{*}{ Itens } & \multicolumn{3}{|c|}{ Fatores } \\
\hline & $\begin{array}{c}1 \\
\text { Fazer dieta } \\
\text { e purga } \\
\alpha=.77\end{array}$ & $\begin{array}{c}2 \\
\text { Controle oral } \\
\text { por pressão } \\
\text { social } \\
\alpha=.64 \\
\end{array}$ & $\begin{array}{c}3 \\
\text { Preocupação } \\
\text { com a comida } \\
\alpha=.60\end{array}$ \\
\hline \multicolumn{4}{|l|}{5} \\
\hline 6 & .427 & & \\
\hline 7 & .483 & & \\
\hline 8 & & .751 & \\
\hline 9 & .360 & & \\
\hline 10 & .545 & & \\
\hline 11 & .618 & & \\
\hline 12 & .549 & & \\
\hline 13 & & .663 & \\
\hline 14 & .562 & & \\
\hline 15 & & .409 & \\
\hline 16 & .572 & & \\
\hline 17 & .487 & & \\
\hline 18 & & & .427 \\
\hline \multicolumn{4}{|l|}{19} \\
\hline 20 & & .734 & \\
\hline 21 & & & .812 \\
\hline 22 & .468 & & \\
\hline 23 & .587 & & \\
\hline 24 & .461 & & \\
\hline 25 & & & .387 \\
\hline 26 & .355 & & \\
\hline
\end{tabular}

Fonte: Dados da pesquisa.

0 fator um Fazer dieta e purga faz referência a itens de evitação alimentar e práticas purgatórias. Está composto de 15 itens $(1,2,6,7,9,10,11,12,14,16,17,22$, $23,24,26)$ e com $\alpha=.77$. 0 fator dois Controle oral por pressão social reflete a influência de terceiros sobre o comportamento alimentar da criança. Apresentou quatro itens $(8,13,15,20)$ e $\alpha=.64$, que aumenta a $.720 \mathrm{com}$ a eliminação do item 15. 0 terceiro fator Preocupação com a comida, tem seis itens $(3,4,9,18,21,25)$ e $\alpha=$ .60 , que aumenta a .63 com a eliminação do item 25 e aumenta ainda mais, a .69, se além do 15 , elimina-se o item 18.

Encontraram-se diferenças significativas no primeiro fator Fazer dieta e purga $[F(2,302)=28,879$; $p<0,01]$ segundo o IMC, com os participantes normopeso pontuando menos que os outros em relação a prática de dieta e purgas. Encontram-se diferenças significativas no segundo fator Controle oral por pressão social $[F(2,330)=39,704 ; \mathrm{p}<0,01]$ segundo o IMC, com os participantes normopeso pontuando mais em relação à pressão sentida a comer mais que os outros participantes. Ainda, encontraram-se diferenças significativas no terceiro fator Preocupação com a comida segundo gênero ( $\mathrm{p}<.04)$, com os meninos pontuando mais que as meninas; e segundo a idade, com os alunos de 8 anos pontuando mais que os alunos de outras idades $[F(4,331)=2,49 ; \mathrm{p}<0,05]$.

\section{Atitudes e comportamentos alimentares alterados}

Tomando a pontuação 20 como ponto de corte (Maloney, McGuire \& Daniels, 1988), 43,5\% de amostra da presente investigação estaria em risco a apresentar um problema alimentar, já que apresentam atitudes e comportamentos alimentares alterados ao pontuarem acima de 20 .

A prova $t$ indicou que não existem diferenças significativas entre grupos de gênero. Os meninos apresentaram uma média (desviação típica entre parênteses) $=18.30$ (9.46) e as meninas uma média = 18.93 (9.09). A ANOVA indicou que existem diferenças significativas segundo a idade, com os meninos de 8 anos pontuando mais que os de 10,11 e 12 anos $[F(4,342)=4.88 ; \mathrm{p}=.001]$.

Mais diferenças significativas foram encontradas, segundo o IMC, em atitudes e comportamentos alimentares alterados $[F(2,344)=4.398 ; \mathrm{p}<0.005]$ : os meninos com IMC normal apresentam menos atitudes e comportamentos alimentares alterados que os outros dois grupos.

\section{Discussão}

0 estudo de transtornos alimentares, ou seus sintomas isolados, na infância apresenta duas principais dificuldades: a ausência de um sistema diagnóstico específico para essa idade ou um que contemple aspectos adultos e infantis em separado, já que tanto o DSM-IV quanto o CID-10 são pouco adequados para essa população (Pinheiro, no prelo); e o escasso número de medidas válidas e fiáveis para avaliar tais transtornos nessa idade, obstáculo que aumenta ainda mais quando se trata do idioma português do Brasil. 
Neste país, nos últimos 20 anos, a porcentagem de crianças e adolescentes acima do peso aumentou $240 \%$. Alcançando um índice de $29 \%$ de alunos com sobrepeso e obesidade nas escolas particulares (Coutinho, 1999). Comparando-se com essa investigação, os resultados deste estudo, de $26,8 \%$ de crianças acima do peso, são similares ao da média nacional brasileira. Além disso, o resultado obtido nesta população se aproxima do resultado obtido pela Comissão Europeia: 30\% das crianças europeias entre 7 e 11 anos apresentam peso superior ao adequado a sua idade.

A estrutura fatorial do ChEAT, com três fatores, faz referência à estrutura original proposta pelos autores do EAT-26, Garner, Olmsted, Borh e Garfinkel (1982). Entretanto, a composição de cada fator não coincide exatamente.

0 primeiro fator que aparece, representa Fazer dieta e purga. Está composto por 15 itens e apresenta uma mescla de itens que variam desde a evitação a determinados tipos de alimentos até a prática de vômitos autoinduzidos. Esse fator assemelha-se com o fator do EAT-26 denominado Fazer dieta encontrado por Garner, Olmsted, Borh e Garfinkel (1982) e com o fator de mesmo nome do ChEAT encontrado por Kelly, Ricciardelli e Clarke (1999) e Smolak e Levine (1994). Esse fator coincide, também, com o encontrado por Rodas (2006) em Málaga, excetuando-se o item 26 que no trabalho em questão aparece no segundo fator.

0 segundo fator descreve Controle oral por pressão social. Os quatro itens que compõem tal fator fazem referência à influência de terceiros sobre a conduta alimentar do indivíduo. Esse fator está composto por itens presentes no fator Controle oral de Garner, Olmsted, Borh e Garfinkel (1982) e coincide em todos os itens com o fator de mesmo nome encontrado por Smolak e Levine (1994). Ainda assim, optou-se pela mudança na denominação do fator já que há outros itens que sugerem controle oral, mas não implicam carga social. Igualmente, esse fator é semelhante ao encontrado por Rodas (2006) com a única exceção do mencionado item 26 que, no citado estudo, saturou no primeiro fator.

0 terceiro fator refere-se à Preocupação com a comida. Está composto por itens que afirmam que o sujeito pensa ou reflete por muito tempo acerca do hábito de comer. Os cinco itens deste fator estão no fator Bulimia e Preocupação com a comida de Garner, Olmsted, Borh e Garfinkel (1982), exceto os que fazem referência a bulimia. Tal fator se assemelha também com o fator de mesmo nome encontrado por Sancho, Asorey, Arija e Canals (2005) e por Smolak e Levine (1994). Comparado com a investigação de Rodas (2006), este fator é similar, mas no estudo em questão não saturaram no fator os itens quatro e cinco.

Dos 26 itens do teste, dois saturaram por abaixo do .30: concretamente os itens cinco e 19. Maloney, McGuire e Daniels (1988) eliminaram o item 19 "Eu me controlo diante dos alimentos", desde a primeira análise por haver obtido saturação adequada em nenhum fator do questionário. 0 item cinco, "Eu corto minha comida em pequenos pedaços", tampouco obteve saturação satisfatória para ser incluído em algum fator em muitas investigações (Lynch \& Eppers-Reynolds, 2005; Rodas, 2006; Smolak \& Levine, 1994; Williamson, DeLany, Bentz, Bray, Champagne, \& Harsha, 1997). A consistência interna do questionário, quando se considera os 26 itens, é de .673. Enquanto que, ao excluir-se os itens cinco e 19, sobe para .69. Esse dado indica que é necessário tomar decisões sobre tais itens após uma análise mais detalhada.

Encontraram-se diferenças significativas nos fatores encontrados para o teste segundo gênero, idade e IMC. Os meninos pontuam mais que as meninas no fator três Preocupação com a comida e os alunos de 8 anos pontuam mais neste mesmo fator. Por outro lado, no fator um Fazer dieta e purga e no fator dois Controle oral por pressão social encontraram-se diferenças significativas segundo IMC: os participantes com sobrepeso e obesidade pontuam mais no fator um e menos no fator dois. Esse resultado sugere um equilíbrio aparente em que os que necessitariam controlar sua dieta alimentar e não sentirem-se influenciados a comer mais são exatamente os que o fazem: meninos e meninas com sobrepeso e obesidade.

\section{Considerações finais}

Os resultados mais relevantes desse estudo são: 1) há uma alta porcentagem de pré-adolescentes que se encontram com sobrepeso e obesidade nessa amostra brasileira; 2) há um importante porcentagem deles que se pode considerar em risco a desenvolver um transtorno alimentar completo; 3) a comunidade brasileira já dispõe de uma primeira versão de um instrumento screening validado para avaliar atitudes e comportamentos alimentares problemáticos e, em consequência, poder filtrar os 
pré-adolescentes em situação de risco. Entretanto, essa investigação foi a primeira tentativa de validar o ChEAT no Brasil. Por isso, sugere-se a realização de futuros estudos no mesmo sentido a fim de lograr uma validação definitiva que dê solução à inclusão ou exclusão daqueles itens cujo papel não fica claro neste estudo. Ademais, o uso de um tamanho amostral maior e uma amostra clínica também seriam interessantes para continuar examinando as propriedades psicométricas deste instrumento.

Sugere-se o uso do questionário de 24 itens, eliminando-se os itens cinco e 19, e seguir com atenção aos itens que, eliminados, aumentam a fiabilidade de cada fator. Além disso, ressalta-se que esta é uma versão experimental do teste, e, portanto, deve-se seguir depurando e aperfeiçoando o questionário (Apêndice).

\section{Referências}

Ackard, D. M., \& Peterson, C. B. (2001). Association between puberty and disordered eating, body image, and other psychological variables. International Journal of Eating Disorders, 29(2), 187-194.

Alonso, A. T. V., Rodriguez, M. A. R., Alonso, J. E. L., Carretero, G. R., \& Martín, M. F. (2005). Eating disorders: Prevalence and risk profile among secondary school students. Social Psychiatry and Psychiatric Epidemiology, 40(12), 980-987.

Anton, S. D., Han, H., Newton, R. L. Jr., Martin, C. K., YorkCrowe, E., Stewart, T. M. et al. (2006). Reformulation of the children's eating attitudes test (ChEAT): Factor structure and scoring method in a non-clinical population. Eating and Weight Disorders, 11(4), 201-210.

Cole, T. J., Belizzi, M. C., Flegal, K. M., \& Dietz, W. H. (2000). Establishing a standard definition for child overweight and obesity worldwide: International survey. British Medical Journal, 320(7244), 1-6.

Coutinho, W. (Coord.). (1999). Consenso Latino-Americano de obesidade. Arquivos Brasileiros de Endocrinologia e Metabologia, 43(1), 21-67.

Eapen, V., Mabrouk, A. A., \& Bin-Othman, S. (2006). Disorders eating attitudes and symptomatology among adolescent girls in the United Arab Emirates. Eating Behaviors, 7(1), 53-60.
Edlund, B., Halvarsson, K., \& Sjoden, P. (1996). Eating behaviors, and attitudes to eating, dieting, and body image in 7-year-old Swedish girls. European Eating Disorders Review, 4(1), 40-53.

Forman-Hoffman, V. (2004). High prevalence of abnormal eating and weight control practices among U.S. highschool students. Eating Behaviors, 5(4), 325-336.

Garner, D. M., Olmsted, M. P., Borh, Y., \& Garfinkel, P. E. (1982). The eating attitudes test: Psychometric features and clinical correlates. Psychological Medicine, 12(4), 871-878.

Gracia, M., Marcó, M., \& Trujano, P. (2007). Factores asociados a la conducta alimentaria e preadolescentes. Psicothema, 19(4), 646-653.

Jones, J. M., Bennett, S., Olmsted, M. P., Lawson, M. L., \& Rodin, G. (2001). Disordered eating attitudes and behaviours in teenaged girls: A school-based study. Canadian Medical Association Journal, 165(5), 547-552.

Kaluski, D. N, Natamba, B. K, Goldsmith, R., Shimony, T., \& Berry, E. M. (2008). Determinants of disordered eating behaviors among Israeli adolescent girls. Eating Disorders, 16(2), 146-159.

Kelly, C., Ricciardelli, L. A., \& Clarke, J. D. (1999). Problem eating attitudes and behaviors in young children. International Journal of Eating Disorders, 25(3), 281-286.

Killen, J. D., Taylor, C. B., Hayward, C. H., Haydel, K. F., Wilson, D. M., Hammer, L., et al. (1996). Weight concerns influence the development of eating disorders: A four-year prospective study. Journal of Consulting and Clinical Psychology, 64(5), 936-940.

Knez, R., Munjas, R., Petrovecki, M., Paucic-Kirincic, E., \& Persic, M. (2006). Disordered eating attitudes among elementary school population. Journal of Adolescent Health, 38(5), 628-630.

Lynch, W. C., \& Eppers-Reynolds, K. (2005). Children's eating attitudes test: Revised factor structure for adolescent girl. Eating and Weight Disorders, 10(4), 222-235.

Maloney, M. J., McGuire, J., \& Daniels, R. (1988). Reliability testing of a children's version of the eating attitudes test. Journal of the American Academy of Child and Adolescent Psychiatry, 27(5), 541-543. 
Maloney, M. J., McGuire, J., Daniels. R., \& Specker, B. S. (1989). Dieting Behavior and eating attitudes in children. Pediatrics, 84(3), 482-489.

Pinheiro, N. P. (2011) Classificação e diagnóstico de transtornos alimentares na infância: nem DSM, nem CID10. Revista Psicologia em Pesquisa, 5(1), 61-67.

Ricciardelli, L. A., \& McCabe, M. P. (2001a). Dietary restraint and negative affect as mediators of body dissatisfaction and bulimic behavior in adolescent girls and boys. Behaviour Research and Therapy, 39, 1317-1328.

Ricciardelli, L. A., \& McCabe, M. P. (2001b). Children's body image concerns and eating disturbances: A review of the literature. Clinical Psychology Review, 21(3), 325-344.

Rodas, C. R. (2006). Estructura factorial del Eating Disorder Inventory (EDI-C) y el Children's Eating Attitudes Test (ChEAT). Tesina final de Master no publicada, Universidad de Málaga, España.

Rolland, K., Farnill, D., \& Griffiths, R. A. (1997). Body figure perceptions and eating attitudes among Australian schoolchildren aged 8 to 12 years. International Journal of Eating Disorders, 21(3), 273-278.

Sancho, C., Asorey, O., Arija, V., \& Canals, J. (2005). Psychometrics characteristics of the children's eating attitudes test in a Spanhish sample. European Eating Disorders Review, 13(5), 338-343. doi: 10.1002/erv.643
Schur, E. A., Sanders, M., \& Steiner, H. (2000). Body dissatisfaction and dieting in young children. International Journal of Eating Disorders, 27(1), 74-82.

Senra, C., Seoane, G., Vilas V., \& Sánchez-Cao, E. (2007). Comparison of 10- to 12-year-old boys and girls using a Spanish version of the children's eating attitudes test. Personality and Individual Differences, 42(6), 947-957.

Shisslak, C. M., Crago, M., McKnight, K. M., Estes, L. S., Gray, N., \& Parnaby, O. G. (1998). Potential risk factors associated with weight control behaviors in elementary and middle school girls. Journal of Psychosomatic Research, 44(3/4), 301-313.

Smolak, L., \& Levine, M. P. (1994). Psychometric properties of the children's eating attitudes test. International Journal of Eating Disorders, 16(3), 275-282.

Tremblay, L., \& Lariviere, M. (2009). The influence of puberty onset, Body Mass Index, and pressure to be thin on disordered eating behaviors in children and adolescents. Eating Behaviors, 10(2), 75-83.

Williamson, D. A., DeLany, J. P., Bentz, B. G., Bray, G. A., Champagne, C. M., \& Harsha, D. W. (1997). Gender and racial differences in eating and social pressure to gain weight among children. Journal of Gender, Culture and Health, 2, 231-238. 


\section{APÊNDICE}

Children's Eating Attitudes Test (ChEAT; Maloney, McGuire e Daniels, 1988)

Número controle:

Nome da escola:

Idade:

Série:

Gênero: ( ) Menina

( ) Menino

Peso:

Altura:

Responda às seguintes perguntas de acordo com o que você faz, sente ou pensa.

\begin{tabular}{|c|c|c|c|c|c|c|}
\hline Pergunta & Sempre & $\begin{array}{l}\text { Quase } \\
\text { Sempre }\end{array}$ & Às vezes & $\begin{array}{c}\text { Poucas } \\
\text { vezes }\end{array}$ & $\begin{array}{l}\text { Quase } \\
\text { nunca }\end{array}$ & Nunca \\
\hline 1. Eu tenho medo de ficar acima do peso. & & & & & & \\
\hline 2. Eu tento não comer quando tenho fome. & & & & & & \\
\hline 3. Eu passo muito tempo pensando em comida. & & & & & & \\
\hline $\begin{array}{l}\text { 4. Eu já comi tanto que pensei que não ia mais } \\
\text { conseguir parar. }\end{array}$ & & & & & & \\
\hline 5. Eu corto minha comida em pequenos pedaço & & & & & & \\
\hline $\begin{array}{l}\text { 6. Eu presto atenção na quantidade de calorias } \\
\text { (energia) que há nos alimentos que como. }\end{array}$ & & & & & & \\
\hline $\begin{array}{l}\text { 7. Eu tento não comer alguns alimentos como } \\
\text { pães, batata e arroz. }\end{array}$ & & & & & & \\
\hline $\begin{array}{l}\text { 8. Eu sinto que as outras pessoas gostariam que } \\
\text { eu comesse mais. }\end{array}$ & & & & & & \\
\hline 9. Eu vomito depois de comer. & & & & & & \\
\hline 10. Eu me sinto culpado(a) depois de comer. & & & & & & \\
\hline 11. Eu penso muito em querer ser mais magro(a) & & & & & & \\
\hline $\begin{array}{l}\text { 12. Quando pratico exercícios, eu penso em que } \\
\text { mar calorias (energia). }\end{array}$ & & & & & & \\
\hline $\begin{array}{l}\text { 13. Outras pessoas acham que eu sou muito } \\
\text { magro(a). }\end{array}$ & & & & & & \\
\hline 14. Preocupa-me ter gordura no meu corpo. & & & & & & \\
\hline $\begin{array}{l}\text { 15. Eu demoro mais tempo que as outras pesso } \\
\text { para terminar de comer minhas refeições. }\end{array}$ & & & & & & \\
\hline $\begin{array}{l}\text { 16. Eu tento não comer alimentos que contenha } \\
\text { açúcar. }\end{array}$ & & & & & & \\
\hline 17. Eu como alimentos dietéticos ou light. & & & & & & \\
\hline 18. Eu acho que a comida controla minha vida. & & & & & & \\
\hline
\end{tabular}




\begin{tabular}{|l|l|l|l|l|l|l|}
\hline \multicolumn{1}{|c|}{ Pergunta } & Sempre & $\begin{array}{c}\text { Quase } \\
\text { Sempre }\end{array}$ & Às vezes & $\begin{array}{c}\text { Poucas } \\
\text { vezes }\end{array}$ & $\begin{array}{c}\text { Quase } \\
\text { nunca }\end{array}$ & Nunca \\
\hline $\begin{array}{l}\text { 19. Eu demonstro autocontrole diante dos } \\
\text { alimentos. }\end{array}$ & & & & & & \\
\hline $\begin{array}{l}\text { 20. Eu sinto que as outras pessoas me pressio- } \\
\text { nam para comer. }\end{array}$ & & & & & & \\
\hline 21. Eu penso muito em comer. & & & & & & \\
\hline 22. Eu me sinto mal depois de comer doces. & & & & & & \\
\hline 23. Eu já fiz regimes para emagrecer. & & & & & & \\
\hline 24. Eu gosto de sentir meu estômago vazio. & & & & & & \\
\hline $\begin{array}{l}\text { 25. Eu gosto de experimentar novas comidas } \\
\text { ricas em calorias (energia). }\end{array}$ & & & & & & \\
\hline 26. Eu sinto vontade de vomitar depois de comer. & & & & & & \\
\hline
\end{tabular}

Obrigada pela sua participação! 